\title{
An alternate energy-conserved pathway for the Morita-Baylis-Hillman (MBH) reaction
}

Veejendra K. Yadav*

Department of Chemistry, Indian Institute of Technology, Kanpur 208016, India

vijendra@iitk.ac.in

Abstract. An new overall lower energy pathway for the amine-catalysed Morita-Baylis-Hillman reaction is proposed from computations at the M06-2X/6-311++G(d,p) level. The pathway involves proton-transfer from the ammonium ion to the alkoxide formed from the aldol reaction through a seven-membered ring transition state (TS) structure followed by highly exothermic Hofmann elimination through a fivemembered ring TS structure to form the product and also release the catalyst to carry on with the process all over again.

Introduction. The $\mathrm{MBH}$ reaction ${ }^{1}$ is generally believed to follow the pathway given in Scheme 1 by taking $\mathrm{Me}_{3} \mathrm{~N}(\mathbf{1})$ as the organocatalyst, cyclopentenone (2) as the activated alkene and acetaldehyde (4) as the aldol partner. Reversible conjugate addition of $\mathrm{Me}_{3} \mathrm{~N}$ to cyclopentenone generates the enolate 3, which enters aldol reaction with the aldehyde $\mathbf{4}$ and forms the zwitter ion $\mathbf{5}$. A proton-transfer to the alkoxide through a four-membered ring transition state (TS) structure generates yet another zwitterion 6a, which promptly undergoes E1cB elimination to form the product $7 .^{2}$

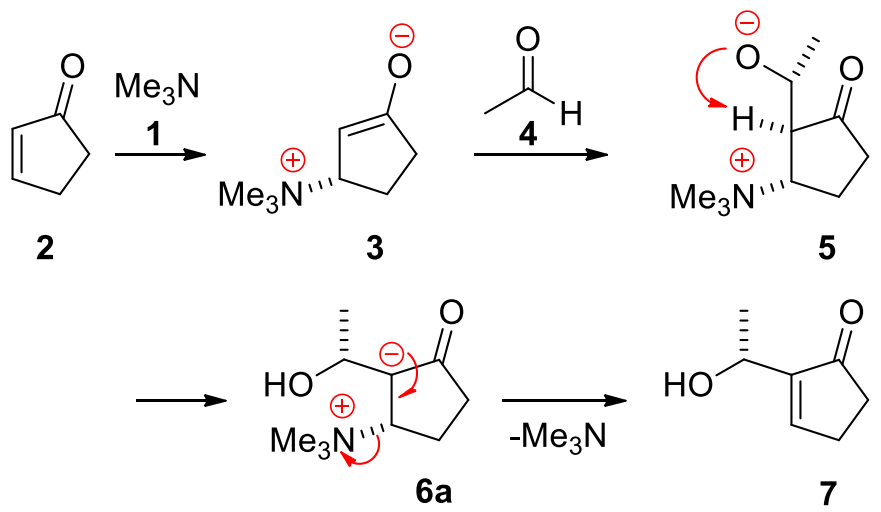

Scheme 1 . The generally accepted pathway for the $\mathrm{Me}_{3} \mathrm{~N}$-catalysed $\mathrm{MBH}$ reaction of cyclopentenone with acetaldehyde 
Hill and Isaacs $^{3}$ suggested the aldol step to be rate-limiting from kinetic studies in 1980s. However, McQuade $^{4}$ and also Aggarwal ${ }^{2 b}$ evaluated the reaction mechanism by kinetic and theoretical means and proposed the proton transfer step $\mathbf{5} \rightarrow \mathbf{6}$ as rate-limiting. Aggarwal also indicated that the intramolecular four-membered ring proton transfer is unlikely because of the strain induced in attaining the appropriate eclipsed conformation in the TS structure. ${ }^{2 \mathrm{~b}}$ Intermolecular proton-transfer between two alkoxide species is also a possibility but not considered till date. In 2015, Singleton and Plata showed that the reaction conditions determined the limiting step of the reaction. ${ }^{2 e}$ Proton-transfer was the primary rate-limiting step at $25{ }^{\circ} \mathrm{C}$, but the aldol step was partially rate-limiting and became the primary rate-limiting step at low temperatures.

We consider a hitherto unexplored plausible pathway for the $\mathrm{MBH}$ reaction and provide the complete potential energy surface from computations at a reasonably high level of theory. The working hypothesis is demonstrated in Scheme 2. The enolate $\mathbf{3}$ formed from conjugate addition of $\mathrm{Me}_{3} \mathrm{~N}$ to cyclopentenone through the TS structure TS1 combines with $\mathrm{CH}_{3} \mathrm{CHO}$ in aldol fashion through the TS structure resembling TS2 and generates the zwitterion species $\mathbf{5}$. The alkoxide function in $\mathbf{5}$ abstracts a proton from a methyl group on $\mathrm{N}$ through the seven-membered ring TS structure resembling TS3 and forms the ylide $\mathbf{6 b}$. Now, Hofmann syn elimination ${ }^{5}$ as shown in TS4 delivers the MBH product 7. TS2 is conceived by avoiding possible steric interactions in the anti-TS conformation. ${ }^{6}$

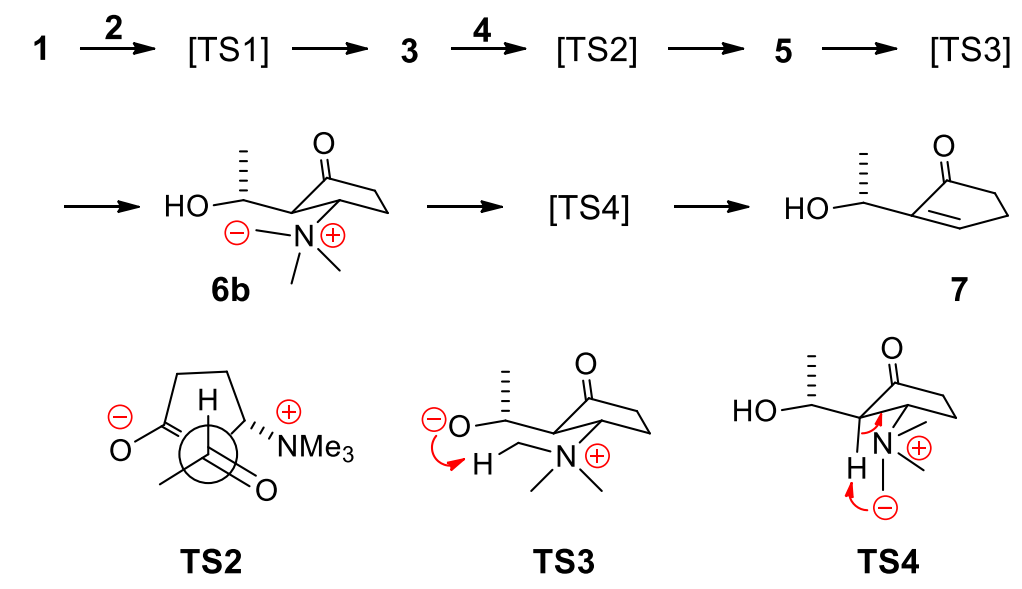

Scheme 2. The conceived reaction pathway for the $\mathrm{MBH}$ reaction of cyclopentenone and acetaldehyde in the presence of trimethylamine as the catalyst 
Computational methods: All the structures, geometry optimizations and TS structure searches were carried out using the global hybrid meta-GGA M06-2X density functional and 6-311++G(d,p) basis set at 298.15 K. ${ }^{7}$ The optimized structures were verified as minima or first order saddle points on their potential energy surfaces by harmonic vibrational frequency analysis. Calculations were carried out using the Gaussian 16 suite of programs. ${ }^{8}$

Results and Discussion. The choice of cyclopentenone as the activated alkene was guided by the requirement of limiting the conformational flexibility of the enolate formed from conjugate addition of the amine. Since it has been amply demonstrated in the literature that the rate-limiting step is either the carbon-carbon bond formation during the aldol step or the proton-transfer in the subsequent step or a mix of both depending on the reaction conditions, we concentrated on the proton-transfer step $5 \rightarrow$ TS3 $\rightarrow 6 b$ and the subsequent Hofmann elimination $\mathbf{6 b} \rightarrow$ TS4 $\rightarrow 7$ to estimate their relative ease of occurrence in comparison to the aldol reaction $(3+4) \rightarrow T S 2 \rightarrow 5$. We have also estimated the ease of the conjugate addition step $(\mathbf{1}+\mathbf{2}) \rightarrow \mathrm{TS} \mathbf{1} \rightarrow \mathbf{3}$ to generate the entire potential energy surface of the reaction as shown in Figure 1.

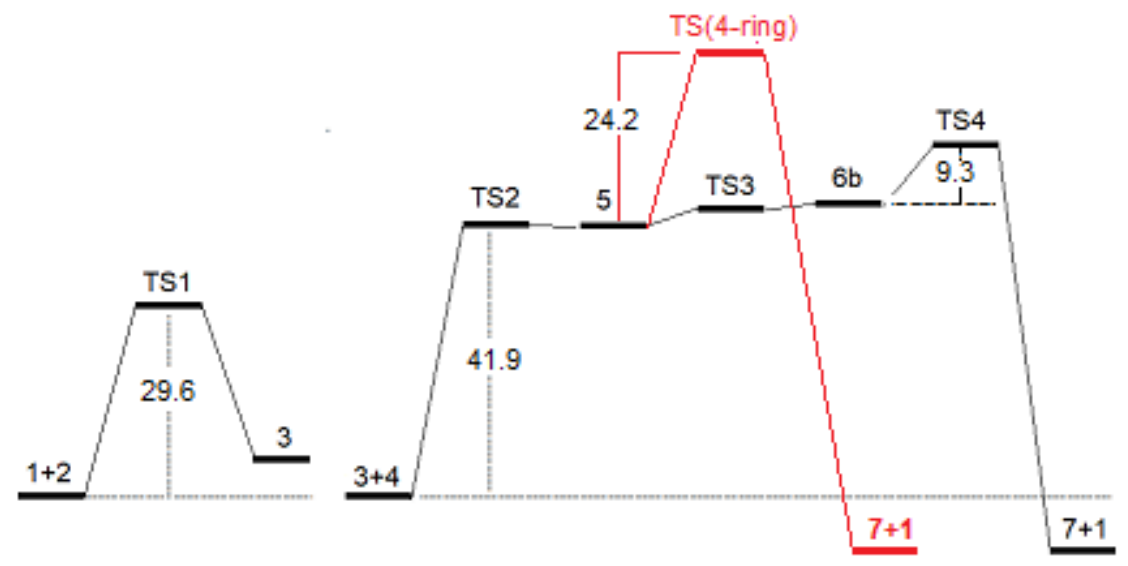

Figure 1. The relative potential energy surface of the gas phase $\mathrm{M}_{3} \mathrm{~N}$-catalysed $\mathrm{MBH}$ reaction of cyclopentenone with acetaldehyde following the pathway proposed in Scheme 2 . TS(4-ring) is TS for the transformation $\mathbf{5} \rightarrow \mathbf{6 a}$.

While the free energy of activation $\left(\Delta \mathrm{G}^{\ddagger}\right)$ of the aldol step $(\mathbf{3}+\mathbf{4}) \rightarrow \mathrm{TS} 2 \rightarrow \mathbf{5}$ is the largest at $41.9 \mathrm{kcal} / \mathrm{mol}$, that of the initial conjugate addition step $(\mathbf{1}+\mathbf{2}) \rightarrow \mathrm{TS} \mathbf{1} \rightarrow \mathbf{3}$, proton transfer step $\mathbf{5} \rightarrow \mathrm{TS} 3 \rightarrow \mathbf{6 b}$ and the elimination step $\mathbf{6 b} \rightarrow \mathbf{T S} 4 \rightarrow \mathbf{7}$ are much lower at $29.6 \mathrm{kcal} / \mathrm{mol}, 2.6 \mathrm{kcal} / \mathrm{mol}$ and $9.3 \mathrm{kcal} / \mathrm{mol}$, respectively. 
Except the last step, all other steps are mildly to sufficiently endothermic. The last step is exothermic by a whopping $52.9 \mathrm{kcal} / \mathrm{mol}$. The exothermic nature of the overall process $(\mathbf{1 + 2 + 4}) \rightarrow(\mathbf{1 + 7})$ by $2.5 \mathrm{kcal} / \mathrm{mol}$ may be responsible for the reaction forward. Clearly, an increase in the reaction temperature is likely to switch on the equilibrium to the reactants occurring at moderate temperatures and make the $\mathrm{MBH}$ reaction less efficient. ${ }^{9}$

The alkoxide-initiated deprotonation of the ammonium ion, $\mathbf{5} \rightarrow \mathbf{T S} 3 \rightarrow \mathbf{6 b}$, is a rapid process for the requirement of only $2.6 \mathrm{kcal} / \mathrm{mol}$ energy. Likewise, the proposed elimination $\mathbf{6 b} \rightarrow \mathbf{T S} 4 \rightarrow \mathbf{7}$ is also a relatively rapid process for the requirement of only $9.3 \mathrm{kcal} / \mathrm{mol}$ energy, easily occurring at $25{ }^{\circ} \mathrm{C}$, in comparison to both the conjugate addition and the aldol reaction. The strong basic character of the alkoxide and the strong acidic character of the methyl-hydrogen in the ammonium group facilitate the deprotonation.

We have also calculated the free energy of activation of the four-membered ring proton-transfer $5 \rightarrow 6 \mathbf{a}$ shown in Scheme 1 to be $24.2 \mathrm{kcal} / \mathrm{mol}$ against the $9.3 \mathrm{kcal} / \mathrm{mol}$ for the five-membered ring protontransfer $\mathbf{6 b} \rightarrow \mathbf{T S} 4 \rightarrow \mathbf{7}$ shown in Scheme 2 . Having noted that the first proton-transfer $\mathbf{5} \rightarrow \mathbf{T S} \mathbf{3} \rightarrow \mathbf{6 b}$ is extremely rapid under the typical $\mathrm{MBH}$ reaction conditions, the pathway shown in Scheme 2 offers an overall lower energy pathway than the generally accepted pathway given in Scheme 1.

It is clear from the potential energy profile in Figure 1 that the aldol step is the most energy demanding and, hence, the slowest in the sequence of reactions. Among the reactions following aldol, the elimination $\mathbf{6 b} \rightarrow$ TS4 $\rightarrow \mathbf{7}$ involving proton transfer is the slowest and, hence, it may possibly be considered to have scope for $k_{\mathrm{H}} / k_{\mathrm{D}}$ kinetic isotope effect. The $k_{\mathrm{H}} / k_{\mathrm{D}}$ was calculated to be 1.26 from the ratio of the single negative frequencies of the respective TS structures. ${ }^{10}$

We have also calculated the profile of the $\mathrm{Me}_{3} \mathrm{~N}$-catalysed $\mathrm{MBH}$ reaction of acrylonitrile $\left(\mathrm{CH}_{2}=\mathrm{CHCN}\right)$ with acetaldehyde as in Figure 2. This profile is much similar to that of the $\mathrm{Me}_{3} \mathrm{~N}$-catalysed reaction of cyclopentenone with acetaldehyde. Since the activation energies of the proton transfers through the seven-membered $(2.2 \mathrm{kcal} / \mathrm{mol})$ and also the subsequent Hofmann elimination $(6.5 \mathrm{kcal} / \mathrm{mol})$ are small in comparison to those of the four-membered ring TS structure $(23.3 \mathrm{kcal} / \mathrm{mol})$, conjugate addition $(26.1$ $\mathrm{kcal} / \mathrm{mol}$ ) and aldol reaction $(36.1 \mathrm{kcal} / \mathrm{mol})$, no $k_{\mathrm{H}} / \mathrm{k}_{\mathrm{D}}$ kinetic isotope effect will be expected to be seen. The calculated $k_{H} / k_{\mathrm{D}}$ isotope effect is 1.35 for the Hofmann elimination step and 1.37 for the four- 
membered ring proton-transfer step. In confirmation of the computational results, Hill and Isaacs have experimentally observed no kinetic isotope effect for the measured $k_{H} / k_{D}$ as $1.03 \pm 0.1$ for the reaction of $\alpha-{ }^{2} \mathrm{H}$ acrylonitrile $\left(\mathrm{CH}_{2}=\mathrm{CDCN}\right)$ with acetaldehyde under catalysis by 1,4-diazabicyclo[2.2.2]octane (DABCO). ${ }^{3 \mathrm{C}}$

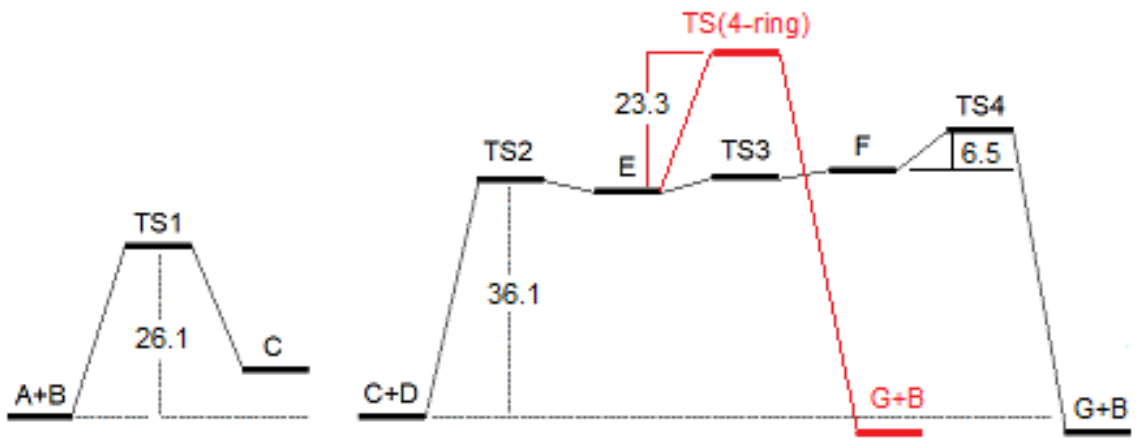

Figure 2. The relative potential energy surface of the gas phase $\mathrm{M}_{3} \mathrm{~N}$-catalysed $\mathrm{MBH}$ reaction of acrylonitrile with acetaldehyde. TS(4-ring) is TS for the transformation equivalent to $5 \rightarrow 6 \mathbf{a}$. $[A=$ acrylonitrile, $\mathrm{B}=\mathrm{Me}_{3} \mathrm{~N}, \mathrm{C}=$ conjugate addition product of $\mathrm{A}$ and $\mathrm{B}, \mathrm{D}=\mathrm{CH}_{3} \mathrm{CHO}, \mathrm{E}=$ aldol product (the alkoxide), TS3 = TS for proton transfer from the ammonium group in $\mathrm{E}$ to the alkoxide, $\mathrm{F}=$ ammonium ylide, TS4 = TS for Hofmann elimination, $\mathrm{G}=$ MBH product $]$

Conclusion. We have presented an alternate lower energy route for the gas-phase amine-catalysed MBH reaction that involves (a) proton-transfer from the alkyl group of the ammonium species to the alkoxide formed from aldol reaction through a seven-membered ring TS structure to generate an ammonium ylide, $5 \rightarrow$ TS3 $\rightarrow$ 6b, and (b) Hofmann elimination of the elements of the amine through a five-membered ring TS structure to form the $\mathrm{MBH}$ product, $6 \mathrm{~b} \rightarrow \mathrm{TS} 4 \rightarrow \mathbf{7}$, and also regenerate the amine to carry on with its catalytic role. Several MBH reactions under solvent-less conditions have been reported in the literature and, at times, found to be faster and also better yielding than those in solvents. ${ }^{11}$ The study of the influence of solvents ${ }^{12}$ on the overall reaction profile and also the kinetic isotope effect $k_{\mathrm{H}} / k_{\mathrm{D}}$ is currently under investigation.

\section{ASSOCIATED CONTENT}

\section{Supporting Information}


Supporting Information (SI) available: Cartesian coordinates of the optimized substrates, transition state structures, Gibbs' free energies of the ground and transition state structure studied at the level M06-2X/6$311++G(d, p)$.

\section{ORCID}

Veejendra K. Yadav: 0000-0001-8294-0677

\section{ACKNOWLEDGEMENTS}

The author acknowledges allocation of time on HPC series of supercomputers by the Computer Centre, Indian Institute of Technology Kanpur.

\section{REFERENCES}

1. (a) Morita, K.; Suzuki, Z.; Hirose, H. A Tertiary Phosphine-catalyzed Reaction of Acrylic Compounds with Aldehydes. Bull Chem Soc. Jpn. 1968, 41, 2815-2816. (b) Morita, K.; Kobayashi, T. New Addition Reactions of Acrylic Compounds with Fumaric Acid Esters Catalyzed by Tervalent Phosphorus Compounds. Bull Chem Soc Jpn. 1969, 42, 2732-2732. (b) Baylis, A. B.; Hillman, M. E. D. German Patent 1972, 2155113; Chem. Abstr. 1972, 77, 34174q.

2. (a) Basavaiah, D.; Rao, A. J.; Satyanarayana, T. Recent Advances in the Baylis-Hillman Reaction and Applications. Chem. Rev. 2003, 103, 811-891. (b) Aggarwal, V. K.; Fulford, S. Y.; Lloyd-Jones, G. C. Reevaluation of the Mechanism of the Baylis-Hillman Reaction: Implications for Asymmetric Catalysis. Angew. Chem. Int. Ed. 2005, 44, 1706-1708. (c) Buskens, P.; Klankermayer, J.; Leitner, W. Bifunctional Activation and Racemization in the Catalytic Asymmetric Aza-Baylis-Hillman Reaction. J. Am. Chem. Soc. 2005, 127, 16762-16763. (d) Robiette, R.; Aggarwal, V. K.; Harvey, J. N. Mechanism of the Morita-Baylis-Hillman Reaction: A Computational Investigation. J. Am. Chem. Soc. 2007, 129, 15513-15525. (e) Plata, R. E.; Singleton, D. A. A Case Study of the Mechanism of Alcohol-Mediated Morita Baylis-Hillman Reactions. The Importance of Experimental Observations. J. Am. Chem. Soc. 2015, 137, 3811-3826. (f) Pellissier, H. Recent developments in the asymmetric organocatalytic Morita-Baylis-Hillman reaction. Tetrahedron 2017, 73, 28312861.

3. (a) Hill, J. S.; Isaacs, N. S. Functionalisation of the $\alpha$ position of acrylate systems by the addition of carbonyl compounds: Highly pressure-dependent reactions. Tetrahedron Lett. 1986, 27, 50075010. (b) Hill, J. S.; Isaacs, N. S. Mechanism of $\alpha$-Substitution Reactions of Acrylic Derivatives. J. Phys. Org. Chem. 1990, 3, 285-288. 
4. (a) Price, K. E.; Broadwater, S. J.; Jung, H. M., McQuade, D. T. Baylis-Hillman Mechanism: A New Interpretation in Aprotic Solvents. Org. Lett. 2005, 7, 147-150. (b) Price, K. E.; Broadwater, S. J.; Walker, D. T; McQuade, D. T. A New Interpretation of the Baylis-Hillman Mechanism. J. Org. Chem. 2005, 70, 3980-3987.

5. (a) Cope, A. C.; Mehta, A. S. Mechanism of the Hofmann Elimination Reaction: An Ylide Intermediate in the Pyrolysis of a Highly Branched Quaternary Hydroxide J. Am. Chem. Soc. 1963, 85, 1949-1952. (b) Bartsch, R. A.; Zavada, J. Stereochemical and base species dichotomies in olefin-forming E2 eliminations. Chem. Rev. 1980, 80, 453-494.

6. (a) Denmark, S. E.; Lee, W. Investigations into Transition-State Geometry in the Mukaiyama Directed Aldol Reaction. Chem. Asian J. 2008, 3, 327-341. (b) Brzezinski, L. J.; Rafel, S.; Leahy, J. W. The Asymmetric Baylis-Hillman Reaction. J. Am. Chem. Soc. 1997, 119, 4317-4318.

7. Zhao, Y.; Truhlar, D. G. The M06 suite of density functionals for main group thermochemistry, thermochemical kinetics, noncovalent interactions, excited states, and transition elements: two new functionals and systematic testing of four M06-class functionals and 12 other functionals. Theor. Chem. Acc. 2008, 120, 215-241.

8. Gaussian 16, Revision C.01, Frisch, M. J.; Trucks, G. W.; Schlegel, H. B.; Scuseria, G. E.; Robb, M. A.; Cheeseman, J. R.; Scalmani, G.; Barone, V.; Petersson, G. A.; Nakatsuji, H.; Li, X.; Caricato, M.; Marenich, A. V.; Bloino, J.; Janesko, B. G.; Gomperts, R.; Mennucci, B.; Hratchian, H. P.; Ortiz, J. V.; Izmaylov, A. F.; Sonnenberg, J. L.; Williams-Young, D.; Ding, F.; Lipparini, F.; Egidi, F.; Goings, J.; Peng, B.; Petrone, A.; Henderson, T.; Ranasinghe, D.; Zakrzewski, V. G.; Gao, J.; Rega, N.; Zheng, G.; Liang, W.; Hada, M.; Ehara, M.; Toyota, K.; Fukuda, R.; Hasegawa, J.; Ishida, M.; Nakajima, T.; Honda, Y.; Kitao, O.; Nakai, H.; Vreven, T.; Throssell, K.; Montgomery, J. A., Jr.; Peralta, J. E.; Ogliaro, F.; Bearpark, M. J.; Heyd, J. J.; Brothers, E. N.; Kudin, K. N.; Staroverov, V. N.; Keith, T. A.; Kobayashi, R.; Normand, J.; Raghavachari, K.; Rendell, A. P.; Burant, J. C.; Iyengar, S. S.; Tomasi, J.; Cossi, M.; Millam, J. M.; Klene, M.; Adamo, C.; Cammi, R.; Ochterski, J. W.; Martin, R. L.; Morokuma, K.; Farkas, O.; Foresman, J. B.; Fox, D. J. Gaussian, Inc., Wallingford CT, 2016.

9. Cantillo, D.; Kappe, C. O. A Unified Mechanistic View on the Morita-Baylis-Hillman Reaction: Computational and Experimental Investigations. J. Org. Chem. 2010, 75, 8615-8626.

10. The $k_{\mathrm{H}} / k_{\mathrm{D}}$ for the proton-transfer step $\mathbf{5} \rightarrow \mathbf{6}$ a was estimated to be 1.36 .

11. (a) Mack, J.; Shumba, M. Rate enhancement of the Morita-Baylis-Hillman reaction through mechanochemistry. Green Chem., 2007, 9, 328-330. (b) Saikia, M.; Sarma, J. C. Baylis-Hillman reaction under solvent-free conditions-Remarkable rate acceleration and yield enhancement 
Can. J. Chem., 2010, 88, 1271-1276. (c) R.-Moghadam, K.; Y.-Miri, L. Quick and efficient synthesis of Morita-Baylis-Hillman adducts of isatin derivatives ARKIVOC 2011 (xi) 43-50. (d) Zhao, S.; Zhi, H.; Zhang, M.; Yan, Q.; Fan, J.; Guo, J. Morita-Baylis-Hillman reaction in eutectic solvent under aqueous medium RSC Adv. 2016, 6, 62778-62784.

12. The medium and the nature of the aldehyde have been reported to have great influence on the absolute value of the kinetic isotope effect for the $\alpha$-position. For instance, the kinetic isotope effect $k_{H} / k_{D}$ was measured at $2.6 \pm 0.1(5.2 \pm 0.6)$ and $1.0 \pm 0.1(2.4 \pm 0.1)$, respectively, in DMSO and THF for the reaction of benzaldehyde ( $p$-nitrobenzaldehyde) and $\alpha^{-2} \mathrm{H}$-labelled methyl acrylate in the presence of DABCO (see ref $2 \mathrm{~d}$ ). 


\section{Table of Contents Graphic}

The amine-catalysed Morita-Baylis-Hillman reaction proceeding through proton transfer through a sevenmembered ring transition state (TS) structure in the aldol species followed by Hofmann elimination through a five-membered ring TS structure is significantly lower in energy than the previously accepted pathway proceeding through proton transfer through a four-membered ring TS structure.

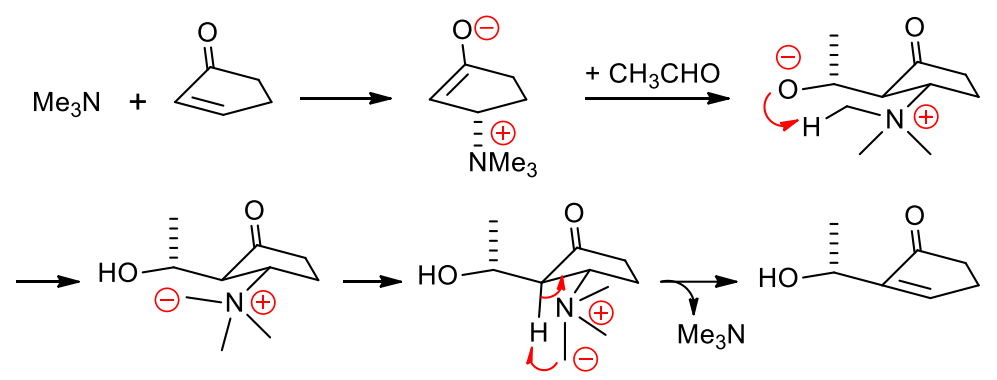

KEYWORDS. Morita-Baylis-Hillman reaction, proton-transfer, Hofmann elimination 